\title{
An update on Staphylococcus aureus infective endocarditis from the International Society of Antimicrobial Chemotherapy
}

Kordo Saeed,

Abhijit BAL

NHS AYRSHIRE AND ARRAN

Ian $M$ Gould

(Medical Microbiology, Aberdeen Royal Infirmary, Aberdeen);

Michael Z. David, M.D., Ph.D.

Department of Medicine, University of Pennsylvania, Philadelphia, Pennsylvania, USA

Matthew Dryden

Efthymia Giannitsioti, MD, PhD

ID consultant, Department of Internal Medicine, NKUA

ATTIKON University General Hospital, Athens, Greece

Karolin Hijazi

(Institute of Dentistry, School of Medicine, Medical Sciences and Nutrition, University of Aberdeen)

Jessica A. Meisner, M.D., M.S.

Department of Medicine, University of Pennsylvania, Philadelphia, Pennsylvania, USA

Esposito Silvano

Department of Infectious Diseases, University of Salerno, Italy

Francesco Scaglione

Department of Oncology and Hemato-oncology, University of Milan, Italy

Pierre Tattevin

Infectious Diseases and Intensive Care Unit, Pontchaillou University Hospital, Rennes, France

Andreas Voss

\section{Introduction}

Staphylococcus aureus remains one of the dominant pathogens in infective endocarditis (IE) causing $25 \%$ to $30 \%$ of all cases $[1,2]$ including healthcare-associated IE which comprises about $30 \%$ of IE [3]. Most IE involves the aortic or mitral valves, with tricuspid valve involvement accounting for fewer than $10 \%$ of cases, often in association with injection drug use $[1,4,5]$. Prosthetic valve IE (PVE) and IE related to cardiovascular implantable electronic devices accounts for approximately one-third of all cases and is most commonly caused by coagulase-negative staphylococci $[1,5]$. 
However, $S$. aureus is equipped with microbial surface components recognizing adhesive matrix molecules (MSCRAMMS): well defined adhesion molecules on the bacterial surface, able to promote adherence to cardiac endothelial cells. S. aureus can multiply and persist within cardiac cells, further promoting vegetation growth via activation of cytokines and thrombotic pathways [6]. Moreover in S. aureus -PVE, surface biofilm formation complicates eradication of the infection. $S$. aureus has been identified as an independent predictor of poor outcome in IE [7,8]. In this review an expert group from the International Society of Antimicrobial Chemotherapy (ISAC) will present recent data, evidence and personal experience on $S$. aureus IE.

\section{Recent evidence related to diagnosis of Staphylococcus aureus IE}

Despite advances in medical provision and interdisciplinary approaches to management, diagnostic latency of $S$. aureus IE remains a challenge. For both native valve IE and PVE diagnosis is still based on major and minor modified Duke criteria [7], entailing microbiology and other laboratory results, echocardiography and clinical appearances or features. Liesman et al. recently published a review on laboratory diagnostics in IE, including the importance of culture and histological examination (if a microbial diagnosis has not been established at the time of surgery, including intraoperative valvular tissue), and molecular techniques [8]. Still in clinically suspected cases, traditional blood cultures (at least three sets, properly filled, ideally taken when patient is febrile and/or prior to commencing antimicrobial therapy) remain central to the diagnosis in most cases [4,9]. However, because the time required for blood culture results may delay diagnosis, novel molecular techniques for pathogen detection, identification and antimicrobial susceptibility may be useful in the future [10].

S. aureus bacteraemia (SAB) in patients with prosthetic valves is a strong predictor of PVE [11]. The British Society for Antimicrobial Chemotherapy (BSAC) recommends echocardiography in all patients with $\mathrm{SAB}$, ideally within the first week of treatment or within 24 hours if there is other evidence to suggest IE [9]. Transthoracic echocardiography (TTE) should be primarily performed in each patient with clinical suspicion of IE; the current European guidelines recommend transoesophageal echocardiography (TOE or TEE) in all patients with negative or non-conclusive TTE findings. An oscillating mass compatible with vegetation is not well visualized by TTE in PVE which has a relatively significant lower sensitivity than in native valve IE [12]. If a prosthetic heart valve or an intracardiac device, e.g., pacemaker, is present, a TOE should be performed as first choice. The only situation in which TTE is equal to TOE with respect to sensitivity of IE detection is right heart IE in patients with a good acoustic window [4]. Additionally novel imaging technologies such as white blood cell (WBC) single-photon emission CT (SPECT)/CT and ${ }^{18} \mathrm{~F}-\mathrm{FDG}$ positron emission tomography (PET)/CT are generally recommended for complex clinical situations [4][13], but are not widely used for variety of reasons including cost, availability, and the experience of interpreting cardiologists. 


\section{Recent evidence related to treatment of Staphylococcus aureus IE}

Guidelines for the treatment of IE were updated in 2015, both in America [14] , and in Europe [4]. Treatment of coagulase-negative staphylococci IE follows exactly the same principles, based on drug-susceptibility testing. The first-line backbone treatments are quite similar in both guidelines: intravenous anti-staphylococcal penicillin or cefazolin for methicillin-susceptible staphylococci IE, and intravenous vancomycin or daptomycin for methicillin-resistant staphylococci IE. Likewise, there is a general agreement that we should not systematically use aminoglycosides in the treatment of native valve staphylococcal IE, regardless of the susceptibility to methicillin, for the following reasons: i) the added value of aminoglycosides has never been proven in staphylococcal IE, even when evaluated in randomized studies [15]; and ii) conversely, recent data demonstrated their deleterious effect, even when administered only for a few days [16].

For staphylococcal PVE, both guidelines recommend gentamicin during the first two weeks of treatment: two or three injections/day for Americans [14], and one or two injections/day for Europeans [4], in association with the backbone regimen (see above), and rifampin, that must be prolonged for at least 6 weeks. Of note, daptomycin is not supported for PVE by current guidelines despite available relevant data and experience with this agent [14]. Although the systematic use of gentamicin and rifampin in staphylococcal PVE is based on a low level of evidence, it could be justified by the requirement for highly bactericidal regimens to eradicate staphylococci on biofilms, taking into account that i) rifampin use has been associated with a lower risk of relapses in other foreign-device related staphylococcal infections (e.g., prosthetic joint infections) [17]; and ii) the early bactericidal effect of aminoglycosides may protect from the emergence of rifampin-resistant isolates, especially during the first days of treatment when bacterial inoculum is large, and the effect of the backbone regimen not fully established (especially with slowly bactericidal agent such as vancomycin).

According to large epidemiological studies, methicillin-resistant S. aureus (MRSA) is more prevalent in PVE than in native valve IE. Early PVE $(<1$ year from valve implantation) is more often caused by hospital-acquired MRSA strains, some with reduced susceptibility to vancomycin. Late PVE demonstrates a microbiological profile similar to community-acquired native IE $[14,18]$. Moreover, complications as valve abscess and embolization are more frequent in PVE. Patients with S. aureus PVE need individualized assessment for valve surgery $[14,18]$. The presence of a prosthetic valve is a major predictor of mortality in S. aureus IE. Valve dehiscence, re-operations and inhospital or 6-month mortality is further increased to $30-50 \%$ in patients with MRSA PVE. This difference may also reflect a higher Charlson comorbidity index score and older age in patients with PVE than native valve IE [18].

European guidelines promoted as an alternative the high dose combination of trimethoprim-sulfamethoxazole (TMP/SMX) $(960 \mathrm{mg} /$ day of trimethoprim, 4,800 $\mathrm{mg} /$ day of sulfamethoxazole) for six weeks, in combination with clindamycin $(1,800$ $\mathrm{mg}$ /day) for one week, in the treatment of native valve $S$. aureus IE, regardless of 
methicillin resistance. However, this should be discouraged in light of the two large randomized trials showing that TMP/SMX is inferior to vancomycin in the treatment of SAB. Likewise, European guidelines suggested cefotaxime as an alternative to cefazolin in penicillin-allergic patients with methicillin-susceptible staphylococci IE, but this is probably sub-optimal, in light of different in vitro, experimental, and cohort studies, suggesting that third-generation cephalosporins are significantly less active than cefazolin for methicillin-susceptible staphylococci [19].

Despite these limitations, the updates of these guidelines are useful, enabling the optimization of staphylococcal IE treatment.

\section{Timing of vavlular surgery for Staphylococcus aureus IE}

The optimal time of valvular surgery in patients with S. aureus IE is still unclear [4]. S. aureus is the most common pathogen in right-sided valve IE (RVIE), but surgical treatment is required (5-16\%) much less often than for left-sided valve IE (LVIE) which generally could reach 50\%. Cardiac surgery in RVIE is most often for prosthetic tricuspid valve $S$. aureus IE. For a damaged tricuspid valve in native IE, early valve surgery (EVS) within 7 days with valve repair is preferable to valve replacement, especially in intravenous drug-users (IVDUs) [20].

Indications for surgery in LVIE includes heart failure, para-valvular complications, persistent infection and $S$. aureus PVE, the latter with level of evidence IIaC [4]. S. aureus PVE is often complicated (stroke, valve dehiscence) and might require EVS; however candidates and timing of surgery are not well defined. In a large international cohort of IE, S. aureus IE patients were treated more conservatively than non-S. aureus IE [OR 0.5, 95\% CI (0.37-0.66)] [21]. Mortality was significantly lower in patients treated with both surgery and antibiotics compared with those treated only medically (33.7\% vs $59 \%, \mathrm{p}=0.001)$. However, a propensity score analysis, adjusted for survival bias, failed to demonstrate an overall significant survival benefit at one year of EVS in $S$. aureus PVE [21]. These results corroborate a previous study of S. aureus PVE in which $>50 \%$ of the patients, especially the younger ones without systemic IE complications, were successfully treated only with antibiotics [22].

Regarding the impact of MRSA on prognosis and the outcome of surgery, data are limited. In one study, all patients with MSSA IE without indications for surgery survived, but one third of patients with MSSA IE who were operated upon indication died. Most MRSA patients were treated only with antibiotics (i.e., without surgery), and 6-month mortality was higher among MRSA than MSSA IE cases [23].

In conclusion, optimal timing of surgery in S. aureus IE should be individualized, taking into account the patients' health status, IE complications, LVIE, PVE and evolution of 
the disease under antimicrobial therapy. Overall, surgery is recommended if benefits outweigh the operative risk. A tailored approach regarding timing of surgery, type of valve and surgical techniques is recommended [4].

\section{New Antimicrobials and Their Role in MRSA IE}

Several new antimicrobials active against $S$. aureus from a variety of drug classes have been approved during the past decade. There are reports documenting successful off-label use of the new agents for bacteremia, endocarditis and other invasive infections (Table 1) $[26,27,29-45,28,46,47]$. Further studies are needed to address the efficacy, and cost effectiveness of these new anti MRSA antibiotics in IE and other invasive infections.

\section{Special Considerations for IE in children}

Despite significant advances, IE in children continues to have a high mortality of up to $10 \%$ although this compares favourably with the mortality in adults. While the basic principles of management of IE in the paediatric age group are broadly similar to those in adults, there are some significant differences. For example, TTE has a sensitivity of $80 \%$ in children and so unlike in adults, TOE is rarely indicated [46]. Echocardiogram should always be performed in children with $\mathrm{SAB}$ as a significant proportion of children have IE [47]. Staphylococci and streptococci are the major causes of IE in children. Staphylococci predominate in children without congenital heart disease. For example in a recent epidemiological investigation in Italy, S. aureus emerged as the leading cause of IE in children without predisposing cardiac ailments with an overwhelming majority $(85.7 \%)$ of $S$. aureus isolates being MRSA [48].

The incidence of IE in neonates is increasing as a result of improved diagnostic techniques and enhanced level of intervention such as the use of central venous catheters (CVCs). Septic emboli are common in neonates leading to signs of metastatic illness, such as seizures [49].

$\beta$-lactamase stable penicillins are the mainstay of treatment of staphylococcal IE on native valves with the option of adding gentamicin for the first 3-5 days. For IE caused by MRSA, vancomycin remains the favoured agent with daptomycin as an alternative. High dose daptomycin $(10 \mathrm{mg} / \mathrm{kg}$ once daily instead of the standard $6 \mathrm{mg} / \mathrm{kg}$ ) is recommended in children $\leq 6$ years to compensate for rapid clearance of this agent from the bloodstream [49] although an even higher dosage (12 mg/kg) has been used [50]. Combination of $\beta$-lactamase stable penicillins and vancomycin is an attractive option for staphylococcal infections until MRSA is ruled out particularly in units where prevalence of MRSA is high [46]. The recommended duration of treatment of staphylococcal IE is at least 4 weeks (6 weeks for MRSA and for PVE). As in adults, addition of rifampicin and gentamicin is recommended for PVE [49]. Experience with newer antibiotics is limited although linezolid has been successfully used in a neonate with IE caused by MRSA 
following failure of vancomycin therapy [51]. Dosing strategies for antibiotics may need to be individualized in order to optimize the pharmacokinetic/pharmacodynamics targets. Nichols and colleagues have published a useful commentary in relation to dose optimization in the paediatric age group in patients with IE [52].

\section{Antibiotic prophylaxis to prevent IE}

Recent reviews of endocarditis prophylaxis in the UK and the US [53-55] have challenged the practice of giving antibiotic prophylaxis to prevent IE in a range of invasive, minor procedures and highlighted the frequency of transient bacteraemia in everyday activities such as brushing teeth, the lack of association of many procedures with endocarditis and have questioned the efficacy of antibiotic prophylactic regimens.

The perceived risk of IE among patients with cardiac conditions undergoing interventions is now felt to be minimal, and lower than the risks of severe adverse events, or indeed loss of life, from the adverse events and volume of prophylactic antibiotics used. For example, antibiotic prophylaxis against IE for dental procedures may lead to a greater number of deaths through fatal anaphylaxis than a strategy of no antibiotic prophylaxis, and is not cost effective.

The following cardiac conditions are regarded as being at higher risk of endocarditis: acquired valvular heart disease with stenosis or regurgitation, valve replacement, structural congenital heart disease, previous IE, and hypertrophic cardiomyopathy. Emphasis is now placed on giving patients clear advice on oral hygiene and dental care, awareness of symptoms of IE and the importance of early medical intervention.

Prophylaxis for IE remains a contentious issue. The UK National Institute for Health and Care Excellence (NICE) guideline published in 2008 and revised in 2015 does not recommend antibiotic prophylaxis even for patients at high risk of IE [56]. Prophylaxis with amoxicillin (clindamycin in penicillin allergic patients) is however recommended by the European Society of Cardiology guidelines for patients undergoing high-risk dental procedures [4]. Studies that were conducted following the restriction of antibiotic prophylaxis provided some evidence of increasing incidence of IE, but there was no microbiological information to support the view that oral streptococci were responsible for the rise in number of cases. Amoxicillin, which is the most commonly used prophylactic agent in the dental setting, is not active against a vast majority of isolates of S. aureus and consequently the debate on prophylaxis is mainly relevant to streptococcal infections. Prevention of $S$. aureus IE focuses mainly on healthcare-associated infections rather than those resulting from community dental procedures.

Prevention of SAB is the key to prevent subsequent IE. Preventing healthcare-associated infection goes beyond the confines of antibiotic prophylaxis. Reduction in the incidence 
of CVC-related bacteraemia can be achieved by improving adherence to infection control practices such as hand hygiene and barrier precautions, full coverage of which is beyond the scope of this review. Guidelines for prevention of intravascular catheter related infections have been published [57], Borg et al. found that hospitals with competency programs in insertion of peripheral venous cannulae and CVCs have a lower prevalence of SAB. Root cause analysis of bacteraemia, hand hygiene audits, and antibiotic stewardship all contribute towards lowering of rates of bacteraemia [58].

Vaccines against $S$. aureus have been investigated but failed to demonstrate efficacy in clinical trials. Vaccination is likely to be of benefit in select groups of patients at high risk of bacteraemia and its complications, for example, patients on chronic haemodialysis. Sustained immune response to the V710 vaccine was demonstrated in clinical trials [59]. However, a phase 3 trial of this vaccine did not demonstrate a reduction in the rate of $S$. aureus infections in the vaccine group. There was also higher mortality in the vaccine group compared to the control group in patients who developed $S$. aureus infection [60]. Much additional research is thus necessary to develop an effective vaccine and to determine the appropriate target population.

\section{Role of Panton Valentine leucocidin (PVL) and other toxins in Staphylococcus aureus IE incidence and outcome}

A range of $S$. aureus virulence factors have been implicated in IE. Most studies have proposed a relationship between IE and expression of secreted virulence factor superantigens in S. aureus. Superantigens include staphylococcal enterotoxins (SE), staphylococcal enterotoxins-like (SEl) molecules, and toxic shock syndrome toxin-1 (TSST-1 encoded by $t s t H$ ). While three of these superantigens (TSST-1, SEB and SEC) are associated with toxic shock syndrome, others may primarily contribute to local effects such as vegetation formation in IE. A prospective study showed that MSSA CC30 IE isolates were significantly more likely to contain genes for TSST-1 as well as some staphylococcal enterotoxins (sea, sed, see) and SEl molecules (sei) [61]. The frequency of sed was significantly higher in MRSA compared to MSSA IE isolates in a small retrospective study, notwithstanding the absence of association with mortality [62]. A more recent experimental study has emphasised the role in IE of an operon comprising one SE gene (seg) and SE-like genes (selo, selm, sei, selu, seln, and seg) known as the enterotoxin gene cluster (egc) that is highly prevalent in $S$. aureus [63]. Stach et al. showed that while TSST-1 contributes to both IE and sepsis, the effect of egc superantigens is limited to vegetation formation in IE. Population-based epidemiological studies to further support the role of $S$. aureus superantigens in IE are not available.

Other studies have focused on the role of regulatory networks such as the quorumsensing operon $a g r$ (accessory gene regulator) implicated in induction of expression of virulence factors. In a rabbit model of IE, infection persistence after treatment with vancomycin correlated with early agr activation [64]. However, other experimental studies suggest that agr is not a key regulator of virulence factor gene expression and instead attributed this role to gene regulator MgrA [65]. Epidemiological data are 
required to confirm the importance of these two global regulatory elements in establishment and progression of IE as well as failure of vancomycin therapy. Evidence to support the role of PVL in pathogenesis of $S$. aureus IE is limited to small studies and case series/reports (Table 2). 


\section{Table 1: Summary of some novel antibiotics with potential role in MRSA Infective Endocarditis}

\begin{tabular}{|l|}
\hline \multicolumn{1}{|c|}{ Agents } \\
\hline \multicolumn{1}{|c|}{ Cephalosporins } \\
Ceftaroline and Ceftobiprole; both active against \\
MRSA and are bactericidal with favorable safety \\
profiles. Both are currently only approved \\
[Ceftaroline (FDA) and ceftobiprole (Europe)] for \\
acute bacterial skin and skin structure infections \\
(ABSSSI) and community acquired pneumonia.
\end{tabular}

\section{Lipoglycopeptides}

Over the last decade three new lipoglycopeptides were approved by the FDA for ABSSSI; Telavancin , dalbavancin and oritavancin. The long half-lives and extended dosing regimen of the latter two make them especially appealing for future use in MRSA IE. However, the long half-lives may be an issue for empirical therapy.

\section{Comments including experience in infective endocarditis (IE)}

There have been case reports and series documenting the successful use of ceftaroline for IE [24], including a successful treatment of vancomycinintermediate and daptomycin-non-susceptible S. aureus [25]. Ceftaroline was reported to be noninferior to vancomycin the treatment of MRSA bacteremia in a retrospective study in Michigan [26] , and was studied in a retrospective multicenter cohort of 211 MRSA bacteremia patients [27]. An observational study from France of left-sided IE treated with ceftaroline either as monotherapy or in combination with another agent reported a cure in 5 of 8 patients [28]. Several other case reports are published on successful use of ceftaroline in combination with daptomycin for MRSA, VISA, and daptomycin non susceptible Staphylococcus aureus IE $[25,29,30]$ but this approach requires further study. To our knowledge there are no published reports of ceftobiprole being used for IE therapy.

Telavancin is a bactericidal once-daily agent that achieved a similar cure rate compared with standard therapy in SAB in a small, randomized trial [31]. In a retrospective study of 14 patients with refractory MRSA bacteremia and IE, the response rate to telavancin was similar to daptomycin or vancomycin; 8 subjects survived to hospital discharge, 5 of whom had IE. The 6 who did not survive all had IE, and 5 of these were deemed not to be surgical candidates [32]. Telavancin is relatively contraindicated in patients with renal dysfunction as it is associated with increased mortality in this population.

Dalbavancin is a lipoglycopeptide with once weekly dosing. In a phase 2 study catheter-related MSSA and MRSA bloodstream infections, dalbavancin had a higher success rate than vancomycin [33]. In a recently published paper, the clinical outcomes and safety of dalbavancin as primary and sequential treatment of Gram-positive bacteremia with IE were evaluated in a single center. Clinical success with dalbavancin was high (92.6\%) but in the majority of patients (24/27) dalbavancin was only used after clearance of bacteria from bloodstream [34]. Another recent retrospective study from Spain assessing dalbavancin therapy for a variety of invasive infections, including bacteremia, demonstrated clinical success in $84.1 \%$ of cases and also a reduction in number of hospitalization days and cost [35]. Despite these encouraging results, there was a recent case report documenting failure of therapy in a pregnant patient with MRSA IE after 4 weeks of dalbavancin [36]. The treatment failed due to reinfection with VISA, perhaps due to inadequate dalbavancin exposure. Another reason for a cautious approach to using this drug for IE is that it was not bactericidal in a rabbit model of IE [37].

Oritavancin is another lipoglycopeptide approved for ABSSSI with a very long half-life, ranging from 245 to $393 \mathrm{~h}$. In a recent case series of 17 patients treated with oritavancin for invasive, complicated Gram-positive infections, including 2 patients with MRSA pneumonia, all improved, although 4 had an adverse reaction [38]. In another case series, 7 of 10 patients with invasive bacterial infections treated with oritavancin were cured although only one of these patients had a MRSA infection [39]. A notable recent report documented success of therapy with oritavancin for a case of refractory MRSA IE [40]. Oritavancin thus may be useful for MRSA IE although more data are needed before it is recommended. 


\section{Oxazolidinone}

Tedizolid is the newest oxazolidinone antibiotic approved for ABSSSI. As for linezolid, it is

considered a bacteriostatic, rather than a

bacteriocidal drug and is not likely to become a

first-line agent for bacteremia or IE. However, it has a high oral bioavailability and only requires once daily dosing [41].

\section{Other novel drugs}

There are several new drugs that may be feasible options for MRSA IE treatment in the future,

including delafloxacin and iclaprim.

Abbreviations: ABSSSI, acute bacterial skin and skin structure infection; FDA, U.S. Food and Drug Administration; IE, infective endocarditis; MSSA, methicillin-susceptible Staphylococcus aureus; MRSA, methicillin-resistant Staphylococcus aureus; SAB, Staphylococcus aureus bacteremia; VISA, vancomycin-intermediate Staphylococcus aureus.
Currently, there are no published case reports of its use in the therapy of IE; however, in a 2015 study of a rabbit model of $S$. aureus IE compared tedizolid, daptomycin, and vancomycin. Tedizolid had modest bactericidal in vivo activity but was less active than the other two drugs [42]. Also, the combination of tedizolid and daptomycin in simulated endocardial vegetations suggested an antagonistic relationship that impeded antimicrobial activity [43]. Additional investigation is thus needed before tedizolid is used to treat IE.

Delafloxacin, a fluoroquinolone approved by the FDA in 2017 for the treatment of ABSSSI with activity against MRSA, demonstrates lower MICs

compared with other fluoroquinolones, a higher barrier to resistance, and good activity against biofilms [44]. However, there are no current studies or reports regarding Delafloxacin use in IE or bacteremia.

Iclaprim is a diaminopyrimadine that inhibits bacterial dihydrofolate reductase. The drug completed Phase 3 trials for ABSSSI that demonstrated it was non-inferior to vancomycin and had a favorable safety profile [45]. While iclaprim is still new to the field, future exploration is warranted for indications for invasive infections. 
Table 2: Evidence to support the role of Panton-Valentine leucocidin (PVL) in pathogenesis of S. aureus-related IE

\begin{tabular}{|l|l|l|}
\hline Background & Risk factors & Reference \\
\hline $\begin{array}{l}\text { IE related to PVL+ CA-MRSA was initially } \\
\text { reported in previously five healthy patients }\end{array}$ & $\begin{array}{l}\text { Following development of } \\
\text { furunculosis }\end{array}$ & {$[66]$} \\
\hline $\begin{array}{l}\text { PVL+ MRSA IE, five cases caused by the } \\
\text { USA300 CA-MRSA clone, and two cases of HA- } \\
\text { MRSA }\end{array}$ & Intravenous drug use & {$[67]$} \\
\hline $\begin{array}{l}\text { In a cohort of 131 HIV-infected patients with } \\
\text { S. aureus bacteraemia, patients with USA300 } \\
\text { PVL+ CA-MRSA infections had significantly } \\
\text { greater odds of IE (OR = 2.73) }\end{array}$ & $\begin{array}{l}\text { HIV patients with USA300 } \\
\text { PVL CA-MRSA infections }\end{array}$ & {$[68]$} \\
\hline $\begin{array}{l}\text { A case of IE related to the PVL+ CA-MRSA } \\
\text { ST93 (Queensland) strain }\end{array}$ & $\begin{array}{l}\text { Intravenous drug use in } \\
\text { one out of two cases; the } \\
\text { second case had no } \\
\text { reported risk factors }\end{array}$ & {$[69]$} \\
\hline $\begin{array}{l}\text { The first and only case of IE relating to PVL+ } \\
\text { CA-MSSA (to the best of our knowledge), } \\
\text { reported in a 76-year old man in Japan who } \\
\text { was treated with ceftriaxone and gentamicin } \\
\text { followed by cardiac surgery }\end{array}$ & $\begin{array}{l}\text { None known, apart from } \\
\text { age }\end{array}$ & {$[70]$} \\
\hline
\end{tabular}

Abbreviations: CA-MSSA, community-associated methicillin-susceptible Staphylococcus aureus; CA-MRSA, community-associated methicillinresistant Staphylococcus aureus; HA-MRSA, healthcare-associated methicillin-resistant Staphylococcus aureus; HIV, human immunodeficiency virus; IE, infective endocarditis; OR, odds ratio; PVL+, Panton-Valentine leucocidin positive. 


\section{Conclusion}

$S$. aureus IE is a common clinical infection with a high mortality. In order to prevent infection and decrease mortality from $S$. aureus IE, further research is need to define its molecular epidemiology, importance of bacterial toxins and other virulence factors, modifiable host risk factors, optimal choice of empiric and definitive antimicrobial therapies, and the timing and indications for valvular surgery. While awaiting these research, native valve staphylococcal IE should be treated with a 4-6 week-course of a single agent, intravenous anti-staphylococcal penicillin or cefazolin for methicillinsusceptible staphylococci, and vancomycin or daptomycin for methicillin-resistant staphylococci. For prosthetic valve staphylococcal IE, this must be reinforced with the adjunction of gentamicin during the first two weeks, and rifampin during the whole duration (i.e. 6 weeks). Timing of surgery in S. aureus IE should be individualized. Further studies are also needed regarding the use of ceftobiprole and ceftaroline as single agents in the management IE.

\section{Acknowledgement \\ Funding}

\section{References}

[1] Murdoch DR, Corey GR, Hoen B, Miró JM, Fowler VG, Bayer AS, et al. Clinical Presentation, Etiology, and Outcome of Infective Endocarditis in the 21st Century. Arch Intern Med 2009;169:463. doi:10.1001/archinternmed.2008.603.

[2] Raoult D, Casalta JP, Richet $\mathrm{H}$, Khan M, Bernit E, Rovery C, et al. Contribution of Systematic Serological Testing in Diagnosis of Infective Endocarditis. J Clin Microbiol 2005;43:5238-42. doi:10.1128/JCM.43.10.5238-5242.2005.

[3] Selton-Suty C, Célard M, Le Moing V, Doco-Lecompte T, Chirouze C, lung B, et al. Preeminence of Staphylococcus aureus in Infective Endocarditis: A 1-Year PopulationBased Survey. Clin Infect Dis 2012;54:1230-9. doi:10.1093/cid/cis199.

[4] Habib G, Lancellotti P, Antunes MJ, Bongiorni MG, Casalta J-P, Del Zotti F, et al. 2015 ESC Guidelines for the management of infective endocarditis. Eur Heart J 2015;36:3075-128. doi:10.1093/eurheartj/ehv319.

[5] Fournier P, Thuny F, Richet H, Lepidi H, Casalta J, Arzouni J, et al. Comprehensive Diagnostic Strategy for Blood Culture-Negative Endocarditis: A Prospective Study of 819 New Cases. Clin Infect Dis 2010;51:131-40. doi:10.1086/653675.

[6] Moreillon P, Que Y-A. Infective endocarditis. Lancet 2004;363:139-49. doi:10.1016/S0140-6736(03)15266-X.

[7] Li JS, Sexton DJ, Mick N, Nettles R, Fowler VG, Ryan T, et al. Proposed Modifications to the Duke Criteria for the Diagnosis of Infective Endocarditis. Clin Infect Dis 2000;30:633- 
8. doi:10.1086/313753.

[8] Liesman RM, Pritt BS, Maleszewski JJ, Patel R. Laboratory Diagnosis of Infective Endocarditis. J Clin Microbiol 2017;55:2599-608. doi:10.1128/JCM.00635-17.

[9] Gould FK, Denning DW, Elliott TSJ, Foweraker J, Perry JD, Prendergast BD, et al. Guidelines for the diagnosis and antibiotic treatment of endocarditis in adults: a report of the Working Party of the British Society for Antimicrobial Chemotherapy. J Antimicrob Chemother 2012;67:269-89. doi:10.1093/jac/dkr450.

[10] Poole S, Kidd SP, Saeed K. A review of novel technologies and techniques associated with identification of bloodstream infection etiologies and rapid antimicrobial genotypic and quantitative phenotypic determination. Expert Rev Mol Diagn 2018;18:543-55. doi:10.1080/14737159.2018.1480369.

[11] Tubiana S, Duval X, Alla F, Selton-Suty C, Tattevin P, Delahaye F, et al. The VIRSTA score, a prediction score to estimate risk of infective endocarditis and determine priority for echocardiography in patients with Staphylococcus aureus bacteremia. J Infect 2016;72:544-53. doi:10.1016/j.jinf.2016.02.003.

[12] Habib G, Badano L, Tribouilloy C, Vilacosta I, Zamorano JL, Galderisi M, et al. Recommendations for the practice of echocardiography in infective endocarditis. Eur J Echocardiogr 2010;11:202-19. doi:10.1093/ejechocard/jeq004.

[13] Hoerr V, Franz M, Pletz MW, Diab M, Niemann S, Faber C, et al. S. aureus endocarditis: Clinical aspects and experimental approaches. Int J Med Microbiol 2018. doi:10.1016/J.IJMM.2018.02.004.

[14] Baddour LM, Wilson WR, Bayer AS, Fowler VG, Tleyjeh IM, Rybak MJ, et al. Infective Endocarditis in Adults: Diagnosis, Antimicrobial Therapy, and Management of Complications. Circulation 2015;132:1435-86. doi:10.1161/CIR.0000000000000296.

[15] Ribera E, Gómez-Jimenez J, Cortes E, del Valle O, Planes A, Gonzalez-Alujas T, et al. Effectiveness of cloxacillin with and without gentamicin in short-term therapy for rightsided Staphylococcus aureus endocarditis. A randomized, controlled trial. Ann Intern Med 1996;125:969-74.

[16] Cosgrove SE, Vigliani GA, Campion M, Fowler, Jr. VG, Abrutyn E, Corey GR, et al. Initial Low-Dose Gentamicin for Staphylococcus aureus Bacteremia and Endocarditis Is Nephrotoxic. Clin Infect Dis 2009;48:713-21. doi:10.1086/597031.

[17] Saeed K, Dryden M, Bassetti M, Bonnet E, Bouza E, Chan M, et al. Prosthetic joints: shining lights on challenging blind spots. Int J Antimicrob Agents 2017;49. doi:10.1016/j.ijantimicag.2016.10.015.

[18] Wang A, Athan E, Pappas PA, Fowler VG, Olaison L, Paré C, et al. Contemporary Clinical Profile and Outcome of Prosthetic Valve Endocarditis. JAMA 2007;297:1354. doi:10.1001/jama.297.12.1354.

[19] Tattevin P, Mainardi J-L. Analysis of the 2015 American and European guidelines for the management of infective endocarditis. Médecine Mal Infect 2016;46:406-10. doi:10.1016/j.medmal.2016.05.008.

[20] Hussain ST, Witten J, Shrestha NK, Blackstone EH, Pettersson GB. Tricuspid valve endocarditis. Ann Cardiothorac Surg 2017;6:255-61. doi:10.3978/14141.

[21] Chirouze C, Alla F, Fowler VG, Sexton DJ, Corey GR, Chu VH, et al. Impact of Early Valve Surgery on Outcome of Staphylococcus aureus Prosthetic Valve Infective Endocarditis: Analysis in the International Collaboration of Endocarditis-Prospective Cohort Study. Clin Infect Dis 2015;60:741-9. doi:10.1093/cid/ciu871.

[22] Sohail MR, Martin KR, Wilson WR, Baddour LM, Harmsen WS, Steckelberg JM. Medical Versus Surgical Management of Staphylococcus aureus Prosthetic Valve Endocarditis. Am 
J Med 2006;119:147-54. doi:10.1016/j.amjmed.2005.09.037.

[23] Hill EE, Peetermans WE, Vanderschueren S, Claus P, Herregods M-C, Herijgers P. Methicillin-resistant versus methicillin-sensitive Staphylococcus aureus infective endocarditis. Eur J Clin Microbiol Infect Dis 2008;27:445-50. doi:10.1007/s10096-0070458-2.

[24] Lin JC, Fierer J, Aung G, Johns S, Thomas A, Jahng M, et al. The use of ceftaroline fosamil in methicillin-resistant Staphylococcus aureus endocarditis and deep-seated MRSA infections: a retrospective case series of 10 patients. J Infect Chemother 2013;19:42-9. doi:10.1007/s10156-012-0449-9.

[25] Ho TT, Cadena J, Childs LM, Gonzalez-Velez M, Lewis JS. Methicillin-resistant Staphylococcus aureus bacteraemia and endocarditis treated with ceftaroline salvage therapy. J Antimicrob Chemother 2012;67:1267-70. doi:10.1093/jac/dks006.

[26] Arshad S, Huang V, Hartman P, Perri MB, Moreno D, Zervos MJ. Ceftaroline fosamil monotherapy for methicillin-resistant Staphylococcus aureus bacteremia: a comparative clinical outcomes study. Int J Infect Dis 2017;57:27-31. doi:10.1016/j.ijid.2017.01.019.

[27] Zasowski EJ, Trinh TD, Claeys KC, Casapao AM, Sabagha N, Lagnf AM, et al. Multicenter Observational Study of Ceftaroline Fosamil for Methicillin-Resistant Staphylococcus aureus Bloodstream Infections. Antimicrob Agents Chemother 2017;61:e02015-16. doi:10.1128/AAC.02015-16.

[28] Tattevin P, Boutoille D, Vitrat V, Van Grunderbeeck N, Revest M, Dupont M, et al. Salvage treatment of methicillin-resistant staphylococcal endocarditis with ceftaroline: a multicentre observational study. J Antimicrob Chemother 2014;69:2010-3. doi:10.1093/jac/dku085.

[29] Cunha BA, Gran A. Successful treatment of meticillin-resistant Staphylococcus aureus (MRSA) aortic prosthetic valve endocarditis with prolonged high-dose daptomycin plus ceftaroline therapy. Int J Antimicrob Agents 2015;46:225-6. doi:10.1016/j.ijantimicag.2015.04.006.

[30] Baxi SM, Chan D, Jain V. Daptomycin non-susceptible, vancomycin-intermediate Staphylococcus aureus endocarditis treated with ceftaroline and daptomycin: case report and brief review of the literature. Infection 2015;43:751-4. doi:10.1007/s15010-0150763-0.

[31] Stryjewski ME, Lentnek A, O'Riordan W, Pullman J, Tambyah PA, Miró JM, et al. A randomized Phase 2 trial of telavancin versus standard therapy in patients with uncomplicated Staphylococcus aureus bacteremia: the ASSURE study. BMC Infect Dis 2014;14:289. doi:10.1186/1471-2334-14-289.

[32] Ruggero MA, Peaper DR, Topal JE. Telavancin for refractory methicillin-resistant Staphylococcus aureus bacteremia and infective endocarditis. Infect Dis (Auckl) 2015;47:379-84. doi:10.3109/00365548.2014.995696.

[33] Raad I, Darouiche R, Vazquez J, Lentnek A, Hachem R, Hanna H, et al. Efficacy and Safety of Weekly Dalbavancin Therapy for Catheter-Related Bloodstream Infection Caused by Gram-Positive Pathogens. Clin Infect Dis 2005;40:374-80. doi:10.1086/427283.

[34] Tobudic S, Forstner C, Burgmann H, Lagler H, Ramharter M, Steininger C, et al. Dalbavancin as Primary and Sequential Treatment for Gram-Positive Infective Endocarditis: 2-Year Experience at the General Hospital of Vienna. Clin Infect Dis 2018. doi:10.1093/cid/ciy279.

[35] Bouza E, Valerio M, Soriano A, Morata L, Carus EG, Rodríguez-González C, et al. Dalbavancin in the treatment of different gram-positive infections: a real-life experience. Int J Antimicrob Agents 2018;51:571-7. doi:10.1016/j.ijantimicag.2017.11.008. 
[36] Steele JM, Seabury RW, Hale CM, Mogle BT. Unsuccessful treatment of methicillinresistant Staphylococcus aureus endocarditis with dalbavancin. J Clin Pharm Ther 2018;43:101-3. doi:10.1111/jcpt.12580.

[37] Lefort A, Pavie J, Garry L, Chau F, Fantin B. Activities of dalbavancin in vitro and in a rabbit model of experimental endocarditis due to Staphylococcus aureus with or without reduced susceptibility to vancomycin and teicoplanin. Antimicrob Agents Chemother 2004;48:1061-4. doi:10.1128/AAC.48.3.1061-1064.2004.

[38] Schulz LT, Dworkin E, Dela-Pena J, Rose WE. Multiple-Dose Oritavancin Evaluation in a Retrospective Cohort of Patients with Complicated Infections. Pharmacother J Hum Pharmacol Drug Ther 2018;38:152-9. doi:10.1002/phar.2057.

[39] Stewart CL, Turner MS, Frens JJ, Snider CB, Smith JR. Real-World Experience with Oritavancin Therapy in Invasive Gram-Positive Infections. Infect Dis Ther 2017;6:277-89. doi:10.1007/s40121-017-0156-z.

[40] Ruggero MA, Ziegler MJ, Tebas P, Binkley A, Kelly BJ. Successful Treatment of MethicillinResistant Staphylococcus aureus Vertebral Osteomyelitis With Outpatient Oritavancin Therapy. Infect Dis Clin Pract 2018;26:141-4. doi:10.1097/IPC.0000000000000599.

[41] Zhanel GG, Love R, Adam H, Golden A, Zelenitsky S, Schweizer F, et al. Tedizolid: A Novel Oxazolidinone with Potent Activity Against Multidrug-Resistant Gram-Positive Pathogens. Drugs 2015;75:253-70. doi:10.1007/s40265-015-0352-7.

[42] Chan LC, Basuino L, Dip EC, Chambers HF. Comparative efficacies of tedizolid phosphate, vancomycin, and daptomycin in a rabbit model of methicillin-resistant Staphylococcus aureus endocarditis. Antimicrob Agents Chemother 2015;59:3252-6. doi:10.1128/AAC.04376-14.

[43] Smith JR, Yim J, Rice S, Stamper K, Kebriaei R, Rybak MJ. Combination of Tedizolid and Daptomycin against Methicillin-Resistant Staphylococcus aureus in an In Vitro Model of Simulated Endocardial Vegetations. Antimicrob Agents Chemother 2018;62:AAC.0010118. doi:10.1128/AAC.00101-18.

[44] Jorgensen SCJ, Mercuro NJ, Davis SL, Rybak MJ. Delafloxacin: Place in Therapy and Review of Microbiologic, Clinical and Pharmacologic Properties. Infect Dis Ther 2018;7:197-217. doi:10.1007/s40121-018-0198-x.

[45] Holland TL, O'Riordan W, McManus A, Shin E, Borghei A, File TM, et al. A Phase 3, Randomized, Double-Blind, Multicenter Study To Evaluate the Safety and Efficacy of Intravenous Iclaprim versus Vancomycin for Treatment of Acute Bacterial Skin and Skin Structure Infections Suspected or Confirmed To Be Due to Gram-Positive Pathogens (REVIVE-2 Study). Antimicrob Agents Chemother 2018;62:e02580-17. doi:10.1128/AAC.02580-17.

[46] Dixon G, Christov G. Infective endocarditis in children. Curr Opin Infect Dis 2017;30:25767. doi:10.1097/QCO.0000000000000370.

[47] Valente AM, Jain R, Scheurer M, Fowler VG, Corey GR, Bengur AR, et al. Frequency of Infective Endocarditis Among Infants and Children With Staphylococcus aureus Bacteremia. Pediatrics 2004;115:e15-9. doi:10.1542/peds.2004-1152.

[48] Esposito S, Mayer A, Krzysztofiak A, Garazzino S, Lipreri R, Galli L, et al. Infective Endocarditis in Children in Italy from 2000 to 2015. Expert Rev Anti Infect Ther 2016;14:353-8. doi:10.1586/14787210.2016.1136787.

[49] Baltimore RS, Gewitz M, Baddour LM, Beerman LB, Jackson MA, Lockhart PB, et al. Infective Endocarditis in Childhood: 2015 Update: A Scientific Statement From the American Heart Association. Circulation 2015;132:1487-515. doi:10.1161/CIR.0000000000000298. 
[50] Prabhudesai S, Kanjani A, Nambi PS, Gnanasambandam S, Ramachandran B. Successful Use of High-dose Daptomycin in a Child With Staphylococcus aureus Endocarditis. Pediatr Infect Dis J 2016;35:517-8. doi:10.1097/INF.0000000000000958.

[51] Sung T-J, Kim H-M, Kim M-J. Methicillin-resistant Staphylococcus aureus endocarditis in an extremely low-birth-weight infant treated with linezolid. Clin Pediatr (Phila) 2008;47:504-6. doi:10.1177/0009922807311736.

[52] Nichols KR, Israel EN, Thomas CA, Knoderer CA. Optimizing Guideline-Recommended Antibiotic Doses for Pediatric Infective Endocarditis. Ann Pharmacother 2016;50:423-7. doi:10.1177/1060028016635660.

[53] Gould FK, Elliott TSJ, Foweraker J, Fulford M, Perry JD, Roberts GJ, et al. Guidelines for the prevention of endocarditis: report of the Working Party of the British Society for Antimicrobial Chemotherapy. J Antimicrob Chemother 2006;57:1035-42. doi:10.1093/jac/dkl121.

[54] Wilson W, Taubert KA, Gewitz M, Lockhart PB, Baddour LM, Levison M, et al. Prevention of Infective Endocarditis: Guidelines From the American Heart Association: A Guideline From the American Heart Association Rheumatic Fever, Endocarditis, and Kawasaki Disease Committee, Council on Cardiovascular Disease in the Young, and the Council on Clinical Cardiology, Council on Cardiovascular Surgery and Anesthesia, and the Quality of Care and Outcomes Research Interdisciplinary Working Group. Circulation 2007;116:1736-54. doi:10.1161/CIRCULATIONAHA.106.183095.

[55] Prophylaxis against infective endocarditis Antimicrobial prophylaxis against infective endocarditis in adults and children undergoing interventional procedures n.d.

[56] Cahill TJ, Baddour LM, Habib G, Hoen B, Salaun E, Pettersson GB, et al. Challenges in Infective Endocarditis. J Am Coll Cardiol 2017;69:325-44. doi:10.1016/j.jacc.2016.10.066.

[57] O'Grady NP, Alexander M, Burns LA, Dellinger EP, Garland J, Heard SO, et al. Guidelines for the Prevention of Intravascular Catheter-related Infections. Clin Infect Dis 2011;52:e162-93. doi:10.1093/cid/cir257.

[58] Borg MA, Hulscher M, Scicluna EA, Richards J, Azanowsky J-M, Xuereb D, et al. Prevention of meticillin-resistant Staphylococcus aureus bloodstream infections in European hospitals: moving beyond policies. J Hosp Infect 2014;87:203-11. doi:10.1016/j.jhin.2014.05.003.

[59] Moustafa M, Aronoff GR, Chandran C, Hartzel JS, Smugar SS, Galphin CM, et al. Phase Ila study of the immunogenicity and safety of the novel Staphylococcus aureus vaccine V710 in adults with end-stage renal disease receiving hemodialysis. Clin Vaccine Immunol 2012;19:1509-16. doi:10.1128/CVI.00034-12.

[60] Fowler VG, Allen KB, Moreira ED, Moustafa M, Isgro F, Boucher HW, et al. Effect of an Investigational Vaccine for Preventing Staphylococcus aureus Infections After Cardiothoracic Surgery. JAMA 2013;309:1368. doi:10.1001/jama.2013.3010.

[61] Nienaber JJC, Sharma Kuinkel BK, Clarke-Pearson M, Lamlertthon S, Park L, Rude TH, et al. Methicillin-Susceptible Staphylococcus aureus Endocarditis Isolates Are Associated With Clonal Complex 30 Genotype and a Distinct Repertoire of Enterotoxins and Adhesins. J Infect Dis 2011;204:704-13. doi:10.1093/infdis/jir389.

[62] Chung J-W, Karau MJ, Greenwood-Quaintance KE, Ballard AD, Tilahun A, Khaleghi SR, et al. Superantigen profiling of Staphylococcus aureus infective endocarditis isolates. Diagn Microbiol Infect Dis 2014;79:119-24. doi:10.1016/j.diagmicrobio.2014.03.009.

[63] Stach CS, Vu BG, Merriman JA, Herrera A, Cahill MP, Schlievert PM, et al. Novel Tissue Level Effects of the Staphylococcus aureus Enterotoxin Gene Cluster Are Essential for Infective Endocarditis. PLoS One 2016;11:e0154762. doi:10.1371/journal.pone.0154762. 
[64] Abdelhady W, Chen L, Bayer AS, Seidl K, Yeaman MR, Kreiswirth BN, et al. Early agr activation correlates with vancomycin treatment failure in multi-clonotype MRSA endovascular infections. J Antimicrob Chemother 2015;70:1443-52. doi:10.1093/jac/dku547.

[65] Gupta RK, Alba J, Xiong YQ, Bayer AS, Lee CY. MgrA Activates Expression of Capsule Genes, but Not the $\alpha$-Toxin Gene in Experimental Staphylococcus aureus Endocarditis. J Infect Dis 2013;208:1841-8. doi:10.1093/infdis/jit367.

[66] Bahrain M, Vasiliades M, Wolff M, Younus F. Five cases of bacterial endocarditis after furunculosis and the ongoing saga of community-acquired methicillin-resistant Staphylococcus aureus infections. Scand J Infect Dis 2006;38:702-7. doi:10.1080/00365540500447150.

[67] Haque NZ, Davis SL, Manierski CL, Vager D, Donabedian SM, Perri MB, et al. Infective endocarditis caused by USA300 methicillin-resistant Staphylococcus aureus (MRSA). Int J Antimicrob Agents 2007;30:72-7. doi:10.1016/j.ijantimicag.2007.02.007.

[68] Furuno JP, Johnson JK, Schweizer ML, Uche A, Stine OC, Shurland SM, et al. Communityassociated Methicillin-resistant Staphylococcus aureusBacteremia and Endocarditis among HIV Patients: A cohort study. BMC Infect Dis 2011;11:298. doi:10.1186/14712334-11-298.

[69] Townell NJ, Munckhof WJ, Nimmo G, Bannan A, Holley A, Daniel A, et al. Communityassociated methicillin-resistant Staphylococcus aureus endocarditis 'down under': Case series and literature review. Scand J Infect Dis 2012;44:536-40. doi:10.3109/00365548.2012.664779.

[70] Nakajima H, Dohi K, Tanabe M, Nakamura A, Kanemitsu S, Wada H, et al. Infective Endocarditis Caused by Panton-Valentine Leukocidin-producing Methicillin-susceptible \&lt;i\&gt;Staphylococcus aureus\&lt;/i\&gt; Identified by the Broad-range PCR Method. Intern Med 2016;55:1871-5. doi:10.2169/internalmedicine.55.5403. 